\title{
Control of Mycobacterium fortuitum and Mycobacterium intracellulare infections with respect to distinct granuloma formations in livers of BALB/C mice
}

\author{
Tânia Regina Marques da Silva, Antonio Luis de Oliveira Almeida Petersen, Theo de Araújo Santos, \\ Taís Fontoura de Almeida, Luiz Antônio Rodrigues de Freitas, Patrícia Sampaio Tavares Veras/+ \\ Centro de Pesquisas Gonçalo Moniz, Fundação Oswaldo Cruz-Fiocruz, Rua Waldemar Falcão 121, 40296-710 Salvador, BA, Brasil
}

\begin{abstract}
Mycobacterium fortuitum is a rapidly growing nontuberculous Mycobacterium that can cause a range of diseases in humans. Complications from $\mathrm{M}$. fortuitum infection have been associated with numerous surgical procedures. A protective immune response against pathogenic mycobacterial infections is dependent on the granuloma formation. Within the granuloma, the macrophage effector response can inhibit bacterial replication and mediate the intracellular killing of bacteria. The granulomatous responses of BALB/c mice to rapidly and slowly growing mycobacteria were assessed in vivo and the bacterial loads in spleens and livers from $\mathrm{M}$. fortuitum and Mycobacterium intracellulare-infected mice, as well as the number and size of granulomas in liver sections, were quantified. Bacterial loads were found to be approximately two times lower in $\mathrm{M}$. fortuitum-infected mice than in $\mathrm{M}$. intracellulareinfected mice and $\mathrm{M}$. fortuitum-infected mice presented fewer granulomas compared to $\mathrm{M}$. intracellulare-infected mice. These granulomas were characterized by the presence of $\mathrm{Mac}_{-} 1^{+}$and $\mathrm{CD}^{+}$cells. Additionally, IFN- $\gamma$ mRNA expression was higher in the livers of $\mathrm{M}$. fortuitum-infected mice than in those of $\mathrm{M}$. intracellulare-infected mice. These data clearly show that mice are more capable of controlling an infection with $\mathrm{M}$. fortuitum than $\mathrm{M}$. intracellulare. This capacity is likely related to distinct granuloma formations in mice infected with $\mathrm{M}$. fortuitum but not with $\mathrm{M}$. intracellulare.
\end{abstract}

Key words: Mycobacterium fortuitum - Mycobacterium intracellulare - granuloma - liver - control of infection

Nontuberculous mycobacteria (NTM) include different species of the genus Mycobacterium that do not belong to the Mycobacterium tuberculosis complex. These include both slowly growing [e.g., Mycobacterium avium-intracellulare (MAI)] and rapidly growing (e.g., Mycobacterium fortuitum and Mycobacterium abscessus) species (Runyon 1959). NTM are human opportunistic pathogens and are predominantly acquired from the environment. A large number of NTM species have been recovered from soil, household dust, water, dairy products, cold-blooded animals, vegetation and human faeces (Ho et al. 2006). These species can also colonize surgical equipment and materials, such as endoscopes and solutions (Brown-Elliott \& Wallace 2005).

In humans, NTM are organisms that belong to a heterogeneous group in which each species of bacteria should be studied separately (Alvarez-Uria 2010). These pathogens can cause a range of diseases affecting a variety of tissues, including the lungs, lymph nodes, skin and soft and skeletal tissue. These diseases can also affect the genitourinary systems and cause disseminated infections (Ho et al. 2006, Griffith et al. 2007, Jarzembowski

\footnotetext{
Financial support: CNPq (306672/2008-1)

+ Corresponding author: pveras@bahia.fiocruz.br

Received 14 January 2010

Accepted 15 June 2010
}

\& Young 2008). MAI is primarily a pulmonary pathogen and is the NTM species most commonly associated with human disease (Griffith et al. 2007). Inhalation of this bacterium may cause pulmonary disease, whereas the ingestion of contaminated water may cause a disseminated disease. A cutaneous manifestation can be attributed to direct inoculation, direct contact or disseminated disease (Weitzul et al. 2000). Infections caused by rapidly growing NTM including $M$. fortuitum can appear after surgical procedures, such as liposuction, silicone injection and breast implantation, or after intravenous catheter insertion, exposure to prosthetic material and pacemaker placement (Sungkanuparph et al. 2003, Palwade et al. 2006, Uslan et al. 2006). There is still no defined optimal treatment for NTM infections because these organisms are resistant to the standard antituberculous agents. In addition, susceptibility to anti-mycobacterial agents varies across different NTM species (ATS 1997).

A protective immune response against pathogenic mycobacterial infections depends on the ability of individuals to form organ granulomas. During infection, mycobacteria induce the formation of these organized immune complexes of differentiated macrophages, lymphocytes and other cells, which are critical for the maintenance of the granuloma architecture and for the restriction of the infection. In the centre of the granuloma, macrophages produce a response that can effectively prevent the replication of bacteria and/or mediate the killing of the intracellular pathogen. On the other hand, compromised granuloma formation is accompanied by dissemination. In addition, the course of the infection in individuals that 
develop compromised granulomas changes from chronic to acute, leading to high morbidity and mortality from mycobacterial infections (Ladel et al. 1995).

The static spatial localization of cells within Mycobacterium-induced granulomas has been extensively studied by standard histological and immunohistochemical methods (Bouley et al. 2001). Little is known, however, about the events involving granuloma formation during infection by rapidly growing mycobacteria. Previous studies have shown that granuloma formation could depend on the Mycobacterium properties (van der Sar et al. 2004) and on the genetic background of the host (Orrell et al. 1992). Using a mammalian model, we compared the granulomatous response of BALB/c mice infected with rapidly or slowly growing Mycobacterium. We compared the bacterial load and the number and size of granulomas from M. fortuitum and M. intracellulareinfected mice. In addition, the cellular composition and mRNA expression of regulatory cytokines and inductible nitric oxide synthase (iNOS) at the site of infection were assessed. The two species of environmental mycobacteria induced distinct responses in BALB/c mice. $M$. fortuitum-infected mice efficiently controlled the bacteria while $M$. intracellulare-infected mice did not. The bacterial load in the spleen and liver, as well, the number of granulomas and the extent of IFN- $\gamma$ mRNA expression in the liver were higher in $M$. fortuitum than in $M$. intracellulare-infected mice.

\section{SUBJECTS, MATERIALS AND METHODS}

Animals - All animal experiments were performed according to the standards of the Oswaldo Cruz Foundation guidelines for animal experimentation and the Committee of Ethics on Animal Experimentation of Gonçalo Moniz Research Center (CPqGM-Fiocruz). Inbred 4-8-week-old female BALB/c mice were obtained from the Animal Facilities Centre of CPqGM-Fiocruz and were maintained under specific pathogen-free conditions.

Bacterial strains and infection - M. intracellulare (ATCC-13950) was obtained from the Prof. Hélio Fraga Reference Center (Rio de Janeiro, Brazil) and M. fortuitum was isolated from naturally infected C57BL/6 mice, as previously described (Da Silva et al. 2002). A bacterial inoculum was prepared by thawing a frozen aliquot of a mycobacterium suspension and diluting it into sterile saline. Mice were injected intravenously via the orbital plexus with a suspension of $10^{7}$ bacteria per mouse in a final volume of $50 \mu \mathrm{L}$. Mice were euthanized at three, seven, 14, 28 and 60 days after infection and the bacterial loads in the infected organs were determined. The liver and spleen were aseptically collected, weighed and homogenized in $7 \mathrm{H} 9$ agar medium (Difco, Detroit, MI, USA) and the bacterial load was determined by plating serial 10 -fold dilutions of tissue homogenates on Middlebrook 7H10 medium (Difco, Detroit, MI, USA) supplemented with OADC (oleic acid, albumin, dextrose, catalase; Becton Dickinson, Germany). The plates were incubated for $2-3$ weeks at $37^{\circ} \mathrm{C}$. Control mice of the same sex and age were injected with sterile saline. The inoculum viability was confirmed by dilution plat- ing of the suspension in duplicate on Middlebrook 7H10 medium (Difco, Detroit, MI, USA) supplemented with OADC. The viability was consistently $80 \%$. Four to five mice per group were euthanized at each time point.

Histopathology - Histological sections from the liver and spleen tissues were collected three, seven, 14, 28 and 60 days after infection. Samples from the liver were fixed in $10 \%$ buffered formalin before paraffin embedding. Sections (4-5 $\mu$ m-thick) were stained with haematoxylin and eosin (H\&E) to evaluate pathological changes, as previously described (Flynn et al. 1998). Granulomas were quantified by counting 10 microscopic fields at 200X magnification per slide. Four to five mice were used per experimental time point. The number of experiments is indicated in the figure legend. Typical clusters of more than six mononuclear cells were characterized as a granuloma. All sections were examined by at least two of the authors in a blinded analysis.

Morphometry - The granuloma size was measured blindly in formalin-inflated livers that were paraffinembedded, sectioned and stained with H\&E. Four to five mice were used per experimental time point. At least 10 lesions per mouse were video-scanned at 400X magnification and the granuloma area was analyzed using the ImagePro 6.0 software after calibration.

Immunohistochemistry - Samples of liver were embedded in Tissue-Tek (Sakura, Zoeterwoude, The Netherlands), immediately frozen on $\mathrm{N}_{2}$ and stored at $-70^{\circ} \mathrm{C}$. The frozen tissues were sectioned ( $6 \mu \mathrm{m}$ thick) on a cryostat (Leica, Nussloch, Germany), air dried and fixed in acetone $\left(10 \mathrm{~min}\right.$ at $\left.4^{\circ} \mathrm{C}\right)$. After a rinse in phosphatebuffered saline, sections were pretreated for $10 \mathrm{~min}$ with $0.003 \% \mathrm{H}_{2} \mathrm{O}_{2}$ and then with avidin and biotin (kit from Dako, Glostrup, Denmark). Sections were stained with a primary antibody against CR3 (Mac-1), CD4, CD8 or B220 for $16 \mathrm{~h}$ at $4^{\circ} \mathrm{C}$. A biotinylated rat anti-IgG antibody (1:100; Sigma, St. Louis, USA) was used as the secondary antibody. The slides were incubated for $1 \mathrm{~h}$ at room temperature (RT), rinsed and incubated in $1 \mu \mathrm{g} / \mathrm{mL}$ streptoavidin-conjugated horseradish peroxidase (Sigma) for 30 min. The slides were then washed and the staining was visualized with a 1:20 dilution of DAB substrate (Dako, Glostrup, Denmark) for 20 min at RT. Finally, the sections were rinsed, counterstained with methyl green and mounted with cover slips. The original magnifications of the photographic image are indicated in the figures legends.

Measurement of $m R N A$ expression by quantitative reverse transcription-polymerase chain reaction ( $q R T$ $P C R)$ - Total RNA was isolated from the liver according to the manufacturer's recommendations using Trizol (Life Technologies, Gaithersburg, USA) and $1 \mu \mathrm{g}$ of total RNA was reverse-transcribed using Superscript II reverse transcriptase (Invitrogen, California, USA). Real-time RT-PCR was performed in optical 96-well reaction plates using SYBR Green PCR Master Mix (Applied Biosystems, Foster City, USA) on an ABI Prism 7900 sequence detection system (Applied Biosystems). The relative level of gene expression was determined 
by the comparative threshold cycle $(\mathrm{Ct})$ method as described by the manufacturer, whereby each sample was normalized to GAPDH expression and the results are expressed as the fold change compared to uninfected controls. The following primer pairs were used: GAPDH, GTTGGTTACAGGCCAGACTTTGTTG (forward) and GAGGGTAGGCTGGCCTATAGGCT (reverse); IFN- $\gamma$, AGAGCCAGATTATCTCTTTCTACCTCAG (forward) and CTTTTTTCGCCTTGCTGCTG (reverse); iNOS, TGCCCCTTCAAGGTTGGTA (forward) and ACTGGAGGGACCAGCCAAAT (reverse); TNF- $\alpha$, AAAATTCGAGTGACAAGCCTGTAG (forward) and CCCTTGAAGAGAACCTGGGAGTAG (reverse) and IL-10, GGTTGCCAAGCCTTATCGGA (forward) and ACCTGCTCCACTGCCTTGCT (reverse).

Statistical analysis - The data are presented using the mean ( \pm standard deviation) values from 3-5 mice per group. All assays were performed three or more times, except the real time RT-PCR, which was performed twice. The statistical analyzes were performed using the Student's $t$-test or the Mann-Whitney test in cases of unequal variances. A $p$ value less than 0.05 was considered significant.

\section{RESULTS}

Course of M. fortuitum and M. intracellulare infection in BALB/c mice - The comparative bacterial loads in the spleens and liver of $M$. fortuitum and $M$. intracellulare-infected mice were assessed. We observed a progressive reduction in the colony forming unit (CFU) count in both the M. fortuitum and M. intracellulareinfected mice; however, the mice infected with M. fortuitum more efficiently controlled the bacteria than the mice infected with $M$. intracellulare. The bacterial load was $1.47 \log$ lower in the spleen and $1.29 \log$ lower in the livers of $M$. fortuitum-infected mice 14 days post-infection than in those of $M$. intracellulare-infected mice ( $\mathrm{p}=0.0079$, Mann-Whitney). At 28 days post-infection, a similar difference was observed: $M$. fortuitum-infected mice had a $1.98 \log$ lower bacterial load in the spleen and a $2.65 \log$ lower level in the liver than M. intracellulareinfected mice ( $\mathrm{p}=0.0159$, Mann-Whitney) (Fig. 1A, B).

Granulomatous response in M. fortuitum and M. intracellulare-infected mice - The numbers of granulomas in the liver sections of M. fortuitum or M. intracellulareinfected mice were compared at the same time points that the bacterial load was determined. At day seven of infection, poorly structured aggregates containing a small number of macrophages and sparse inflammatory infiltrates consisting primarily of lymphocytes were observed in the liver sections of mice infected with either bacterium (Fig. 2A, B). At day 14, the granulomatous response in the liver of $M$. fortuitum-infected mice was characterized by lymphoid aggregates and macrophages, whereas the granulomas consisted primarily of macrophages in M. intracellulare-infected mice (Fig. 2C, D). In addition, in the $M$. intracellulare-infected mice, the portal tract contained a moderate to extensive lymphocytic infiltrate at day 28 after infection (Fig. 2E, F). Overall, the numbers of granulomas in M. fortuitum-
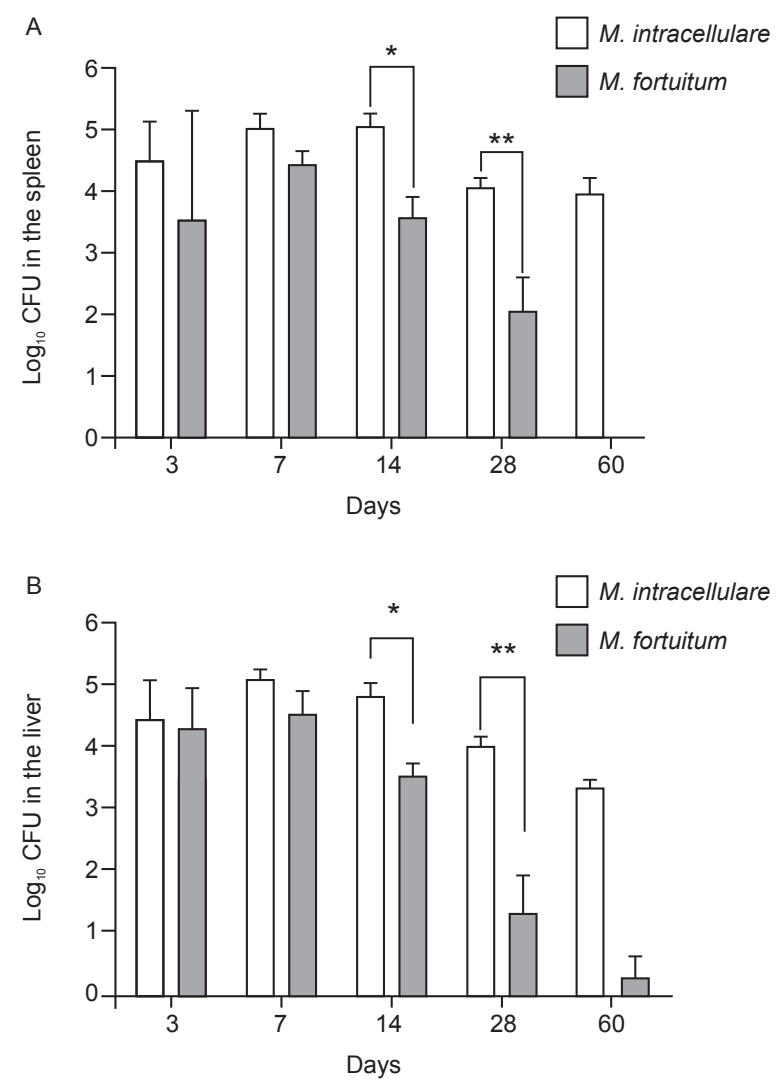

Fig. 1: bacterial load in the spleen or liver from Mycobacterium fortuitum or Mycobacterium intracellulare-infected BALB/c mice. Animals were infected with $10^{7}$ bacteria intravenously (retro-orbital plexus). A: the number of colony forming units (CFU) in the spleen of mice infected with $M$. fortuitum was smaller than the number in mice infected with $M$. intracellulare at all time points examined (*: $\mathrm{p}=0.0079,14$ days; **: $\mathrm{p}=0.0159,28$ days; Mann-Whitney); B: the number of CFUs in the liver of mice infected with M. fortuitum was smaller than the number in mice infected with $M$. intracellulare at all time points examined $(*: \mathrm{p}=0.0079,14$ days; $* *: p=0.0159,28$ days; Mann-Whitney). Each column represents the mean \pm standard error of three ( 3 and 60 days), four ( 7 and 28 days) or five experiments (14 days).

infected mice were five-fold, seven-fold and fifteen-fold lower than the number of granulomas in $M$. intracellulare-infected mice at days 14, 28 and 60 post-infection, respectively $(\mathrm{p}=0.0159$ and $\mathrm{p}=0.0159$ for day 28 and 60 , respectively, Mann-Whitney) (Fig. 2I). We observed a progressive reduction in the granuloma area over time in M. fortuitum-infected mice. In contrast, in $M$. intracellulare-infected mice, the granuloma area enlarged approximately two-fold from day 14-60 after infection. In M. intracellulare-infected mice, the granulomas consisted of tightly grouped epithelioid macrophages in a round structure rimmed by lymphocytes (Fig. 2J).

Immunohistochemistry of granulomatous lesions in M. fortuitum or M. intracellulare-infected mice - The cellular composition of the hepatic granulomas was assessed by immunohistochemistry using specific monoclonal antibodies against macrophages, B cells, $\mathrm{T}$ cells and plasmocytes. Greater numbers of macrophages, 

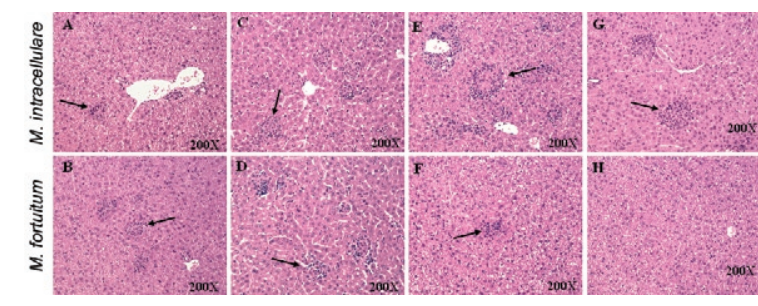

I
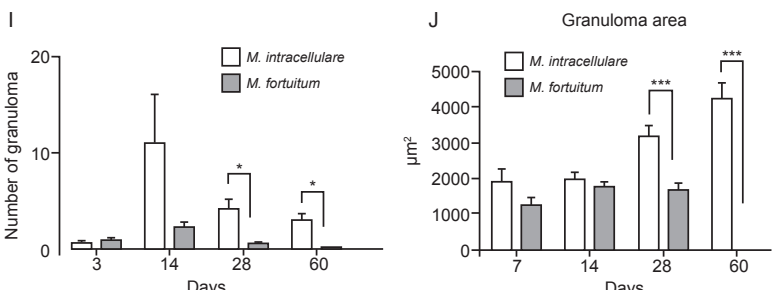

Fig. 2: morphometric analysis of the granuloma present in the liver of BALB/c mice infected with Mycobacterium intracellulare or Mycobacterium fortuitum. Representative images of granulomas (arrow) in liver sections from $M$. intracellulare and $M$. fortuitum-infected mice. After seven (A, B), 14 (C, D), 28 (E, F) or 60 days $(\mathrm{G}, \mathrm{H})$ of infection, animals were euthanized and the liver was fixed in a $10 \%$ formol solution. Liver sections were prepared and stained with haematoxylin and eosin in order to characterize the tissue lesion. I: the granulomas were counted and each column represents the mean of three (7 days), four (14 days) or five experiments (28 and 60 days), containing 3-5 animals per group. A total of 10 fields per lamina per animal were counted. The columns represents the mean number of granulomas per experiment ( $*$ p $=0.0159$; Mann-Whitney); J: the granuloma area were measured and each column represents the mean of five (7 days), 10 (14 and 28 days) and seven sections (60 days). A total of 2-3 sections per experiment were used and the area of 10 granulomas was measured for each section. The data is presented as the mean \pm standard error of granuloma area expressed in $\mathrm{mm}^{2}$ $(* * *: \mathrm{p}=0.0006 ; t$ test $)$.

B cells, $T$ cells and plasmocytes were observed in the M. intracellulare-infected mice than in the M. fortuituminfected mice and the saline-inoculated mice. In both groups of infected mice, granulomas were predominantly composed of Mac-1 $1^{+}$cells (Fig. 3E) and $\mathrm{CD} 4^{+}$cells (Fig. $3 \mathrm{~F})$, in comparison to the other cell types present in the granulomas, such as $\mathrm{CD}^{+}$(Fig. 3G) and B220 (Fig. 3H) cells. By day 14 , the granulomas of $M$. intracellulareinfected mice contained approximately eight times more Mac- $1^{+}$cells and seven times more $\mathrm{CD} 4^{+}$cells than the granulomas in $M$. fortuitum-infected mice. In addition, at day 14 , the numbers of Mac- $1^{+}$cells and $\mathrm{CD}^{+}$cells in M. intracellulare-infected mice were approximately three-fold higher than at seven days post-infection. In contrast, in the $M$. fortuitum-infected mice, we observed an equivalent number of Mac- $1^{+}$cells and $\mathrm{CD} 4^{+}$cells at seven, 14 and 60 days post-infection. On day 28 postinfection, however, the number of $\mathrm{CD}^{+}$cells in the liver granulomas was approximately three-fold higher than the number of $\mathrm{Mac}-1^{+}$cells.

Cytokine expression in the liver of M. fortuitum or M. intracellulare-infected mice - qRT-PCR was used to measure the expression of several cytokines that determine mycobacterial infection outcome (IFN- $\gamma$, TNF- $\alpha$ and IL-
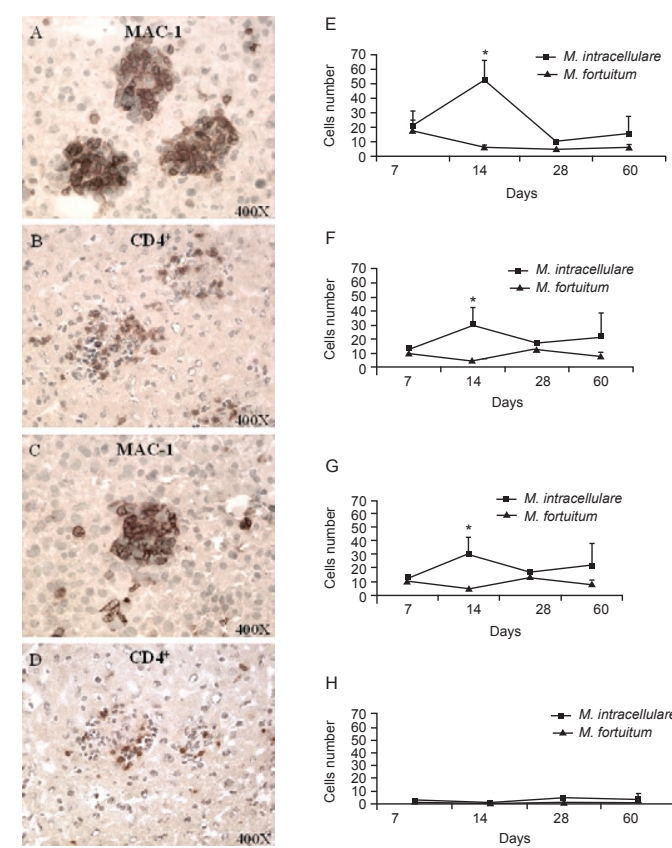

G

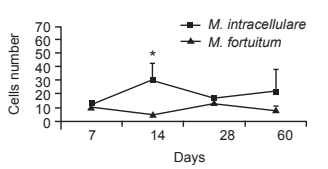

$\mathrm{H}$

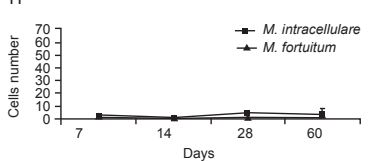

Fig. 3: phenotypic profile of cells in the inflammatory infiltrate in the liver of BALB/c mice infected with Mycobacterium intracellulare or Mycobacterium fortuitum. Representative images of the liver of mice infected with $M$. intracellulare $(\mathrm{A}, \mathrm{B})$ or $M$. fortuitum $(\mathrm{C}, \mathrm{D})$ at 14 days after infection. Note the predominance of Mac- $1^{+}$cells (E) and $\mathrm{CD}^{+} \mathrm{T}$ cells $(\mathrm{F})$ in the inflammatory infiltrate in both groups of infected animals. $\mathrm{CD}^{+}$cells $(\mathrm{G})$ and $\mathrm{B} 220^{+}$cells $(\mathrm{H})$ are present in smaller numbers. In the group infected with $M$. intracellulare, the number of $\mathrm{Mac}-1^{+}$cells starts to decrease at day 14 post-infection (E). On the other hand, in the group infected with $M$. fortuitum the number of $\mathrm{CD}^{+} \mathrm{T}$ cells begin to increase at 28 days post-infection (F). At 14 days post-infection, the group infected with $M$. intracellulare had eight times more Mac- $1^{+}$cells (E) and seven times more $\mathrm{CD}^{+} \mathrm{T}$ cells (F) than the group infected with $M$. fortuitum. The data represent the average number of stained cells \pm standard error $(*: p=0.0286$; Mann-Whitney).

10) and of iNOS, known to be expressed by activated macrophages (MacMicking et al. 1997). This technique was chosen instead of others, such as semi-quantitative non-competitive RT-PCR, northern blot analysis and enzyme linked immunosorbent assay, because it offers several advantages. The most notable is a high degree of sensitivity, allowing for the detection of basal cytokine expression even under experimental conditions in which cytokine production is very low (Hein et al. 2001). qRTPCR was performed using liver samples taken at the time points at which the bacterial load and granuloma morphology were assessed. In both $M$. fortuitum and $M$. intracellulare-infected mice, the mRNA expression levels of TNF- $\alpha$, IL-10 and iNOS were very low and similar to the levels of expression in the control group. At day seven post-infection, the mRNA expression of IFN- $\gamma$ in $M$. fortuitum-infected mice was three-fold higher than in M. intracellulare-infected mice ( $\mathrm{p}=0.0115$, KruskalWallis, Dunn's post-test) (Fig. 4). Similarly, a two-fold increase in IFN- $\gamma$ mRNA expression was observed at day 28 post-infection. 


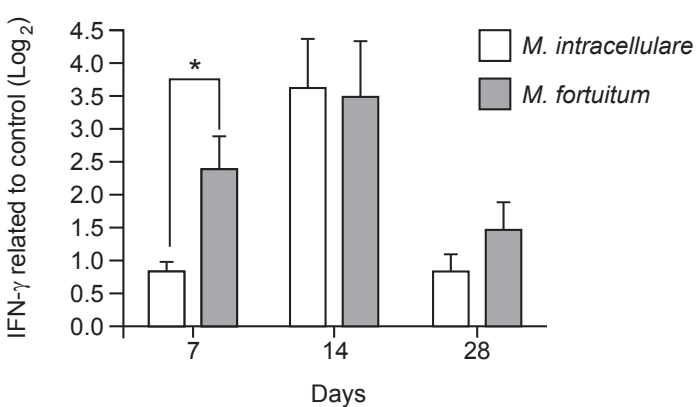

Fig. 4: IFN- $\gamma$ mRNA expression in the liver of BALB/c mice infected with Mycobacterium fortuitum or Mycobacterium intracellulare. The mRNA expression of IFN- $\gamma$ in liver from M. fortuitum-infected mice was three and two times higher than the expression in $M$. intracellulare-infected mice at seven and 28 days post-infection, respectively. The values were normalized to GAPDH expression and were analyzed in relation to the control-uninfected group. The data is presented as the mean ( \pm standard deviation) of one experiment with four animals per group, representative of two experiments performed $(*: \mathrm{p}=0.0115$, Kruskal-Wallis, Dunn's post test).

\section{DISCUSSION}

In the present study, we observed several quantitative and qualitative differences between the granulomatous response of $M$. fortuitum and $M$. intracellulare-infected mice. We observed that $\mathrm{BALB} / \mathrm{c}$ mice more efficiently controlled the $M$. fortuitum infection than the $M$. intracellulare infection. A progressive reduction in the CFU counts was observed in both groups; however, M. fortuitum was almost completely cleared from mice by 60 days post-infection. In contrast, at this time point, bacteria could still be detected in the liver and spleen of $M$. intracellulare-infected mice, although to a lesser extent than at earlier time points. In accordance with these results, a similar approach employed by other authors described a model of BALB/c mice that were intravenously infected with $M$. fortuitum. The authors observed a rapid decrease in the bacterial load in the livers and spleens of infected mice (Parti et al. 2005). However, in the previous study, the authors used a Mycobacterium load in the inoculum that was five-fold greater than that of the present study but observed a complete disappearance of bacterial load from the infected livers after 10 days. This was much faster than was observed in the present study, perhaps due to the different Mycobacterium strains used in the two studies. In agreement with the bacterial load quantification, there were more granulomas in the liver of the $M$. intracellulare-infected mice compared to the M. fortuitum-infected mice (Fig. 2). It is well known that most of the inoculum is trapped in the liver after an intravenous infection (Khalil et al. 1975).

In addition to macrophages, $\mathrm{T}$ cells are important for granuloma formation. T cell-deficient mice do not form granulomas and form loose inflammatory lesions incapable of controlling bacterial growth. The adoptive transfer of $\mathrm{CD}^{+} \mathrm{T}$ cells reconstitutes granuloma formation in RAG-deficient mice infected with Myco- bacterium (Ladel et al. 1995). The $\mathrm{T}$ cell repertoire of a single granuloma is very diverse and similarly sized granulomas in the same organ from the same animal can have different T cell contents in Mycobacterium-infected mice (Hogan et al. 2001). In order to determine the cellular components of the granulomas in the liver of $M$. intracellulare or $M$. fortuitum-infected mice, immunohistological staining was performed using specific antibodies against macrophages, $\mathrm{B}$ cells or T cells. In mice infected with either $M$. intracellulare or M. fortuitum, macrophages and $\mathrm{CD} 4^{+} \mathrm{T}$ cells were the main components of the granulomas. The numbers of macrophages, $\mathrm{CD}^{+}$and $\mathrm{CD} 8^{+} \mathrm{T}$ cells, and $\mathrm{B}$ cells in the liver of M. intracellulare-infected mice were higher than the numbers in the liver of M. fortuitum-infected mice (Fig. 2A-H). These data can be correlated with the higher number and larger size of granulomas observed in the liver of $M$. intracellulare-infected mice (Fig. 2I, J). On day 28 postinfection, the number of $\mathrm{CD}^{+}$cells was approximately three-fold higher than the number of Mac- $1^{+}$cells in the liver of $M$. fortuitum-infected mice. It has been reported that granuloma formation is accelerated by antigen-specific $\mathrm{CD}^{+} \mathrm{T}$ cells and depends, to a large extent, on the TNF- $\alpha$ and IFN- $\gamma$ levels reached within the infected tissues (Appelberg et al. 1994, Hänsch et al. 1996, Chan et al. 2010, unpublished observations).

In order to elucidate the mechanisms underlying the differences in the tissue response induced by $M$. intracellulare and $M$. fortuitum in mice, quantitative RT-PCR for IFN- $\gamma$ and TNF- $\alpha$ was performed on liver samples taken at the same time that the bacterial load and granuloma morphometry were assessed (Fig. 3). At day 24 post-infection, compared to $M$. intracellulare-infected mice, $M$. fortuitum-infected mice expressed more IFN- $\gamma$, which is known to be essential for the control of mycobacterial infection (MacMicking et al. 1997). These data are consistent with the increased number of granulomas and the higher number of $\mathrm{CD}^{+} \mathrm{T}$ cells in the hepatic granulomas. It is likely that the increased number of $\mathrm{CD}^{+} \mathrm{T}$ cells was responsible for the increased IFN- $\gamma$ mRNA level that was observed. Furthermore, the increased expression of IFN- $\gamma$ could explain the accelerated recruitment of inflammatory cells and the increased number of granulomas. We speculate that the observed increase in $\mathrm{CD}_{4}{ }^{+}$cells and IFN- $\gamma$ may be responsible for this enhanced antibacterial response, which leads to the clearance of mycobacteria by 60 days after infection. We expected to detect TNF- $\alpha$ mRNA in the liver tissue of both groups. It has been demonstrated that TNF- $\alpha$-deficient mice have disorganized granulomas following mycobacterial infection, indicating that this cytokine is important for granuloma formation and maintenance (Algood et al. 2005). Unexpectedly, in the liver of mycobacteriuminfected mice, we detected a low TNF- $\alpha$ mRNA level, similar to the level of the control uninfected mice. Previously, it has been demonstrated that the conventional use of an entire infected organ to study granuloma-specific gene expression can yield data that may not genuinely reflect intralesional events (Zhu et al. 2003). In this previous work, the authors identified a downregulation of nine genes that are known to regulate the inflammatory 
response to $M$. tuberculosis, as determined by qRT-PCR analyses of microdissected granuloma-derived cDNA (up to 27-fold) (Zhu et al. 2003). This result points to the idea that use of total liver RNA most likely resulted in the dilution of the gene of interest by nongranulomatous liver tissue, preventing the detection of TNF- $\alpha$ and iNOS mRNA expression in our study.

We did not actually explore the mechanism responsible for the differences in the granulomatous responses of M. fortuitum and M. intracellulare-infected mice. Granuloma formation may depend on the genetic background of the host (Orrell et al. 1992). It has been demonstrated that the Nrampl/SlcllA1 gene exerts a major influence on the early response to infections caused by various atypical mycobacteria such as $M$. fortuitum and M. intracellulare (Denis et al. 1986) by affecting granuloma development (Sato et al. 1990). In addition, Mycobacterium properties (van der Sar et al. 2004) related to differences in pathogen virulence factors, such as cell wall composition, may be responsible for differences in the immune response (Chan et al. 2010, unpublished observations) and probably in granuloma formation. The cell wall of mycobacteria consists of a highly complex array of distinctive lipids, glycolipids and proteins, which are believed to play important roles in the physiology of the bacterium and in the modulation of the host response during infection (Russell et al. 2002, Brennan 2003). Lipoarabinomannans (LAMs) are ubiquitous in mycobacteria and appear to be the most potent non-peptidic cell wall molecules that modulate the host immune response. The LAM structure differs depending on the mycobacterial species. In slow-growing mycobacteria, the arabinan domain is capped by mannose residues (ManLAM) and in rapidly growing mycobacteria, the domain is capped by phosphoinositide motifs (PILAMs) (Chatterjee \& Khoo 1998, Nigou et al. 2002). It has been observed that purified lipids from different strains induce different patterns of cytokines (Mohan et al. 2001). PILAMs more potently induce the secretion of proinflammatory cytokines, such as TNF- $\alpha$ and IL-1, and the production of microbicidal radicals than ManLAMs. In addition, ManLAMs inhibit the IFN- $\gamma$-induced activation of macrophages (Vercellone et al. 1998). These data reinforce the idea that, in our study, the differences observed in the BALB/c mouse responses to either M. fortuitum or $M$. intracellulare infections were related to the composition of the Mycobacterium cell wall.

In summary, this paper compared granuloma formation in $M$. fortuitum and $M$. intracellulare-infected mice. Well-structured granulomas were observed in both groups of infected mice at two weeks after infection as along with a progressive reduction in the bacterial load. Only the group infected with $M$. fortuitum, however, completely cleared the infection and 60 days after infection, the granulomas were resolved, suggesting that the cells in the granulomas were efficiently activated to clear the bacteria. We believe that BALB/c mice are more capable of controlling $M$. fortuitum than $M$. intracellulare infection. Our data reinforce the idea that early pathogen clearance can depend on the mycobacterial species and that some mycobacteria can live within the granuloma in a state of dormancy with the potential to resurface later. While it is generally accepted that granulomas represent a component of the protective immune response against mycobacteria, the precise mechanism underlying the formation and maintenance of granulomas remains to be determined. Understanding the factors that contribute to this long and complex relationship between the pathogen and host is essential to be able to successfully modulate clinical outcomes.

\section{ACKNOWLEDGEMENTS}

To Dr Washington Luis Conrado dos Santos, for valuable advice and help in the statistical analyses used herein, and to Luana Palma, for photographic helpful support.

\section{REFERENCES}

Algood HM, Lin PL, Flynn JL 2005. Tumor necrosis factor and chemokine interactions in the formation and maintenance of granulomas in tuberculosis. Clin Infec Dis 41 (Suppl. 3): S189-193.

Alvarez-Uria G 2010. Lung disease caused by nontuberculous mycobacteria. Curr Opin Pulm Med 16: 251-256.

Appelberg R, Castro AG, Pedrosa J, Silva RA, Orme IM, Minóprio P 1994. Role of gamma interferon and tumor necrosis factor alpha during T-cell-independent and dependent phases of Mycobacterium avium infection. Infect Immun 62: 3962-3971.

ATS - American Thoracic Society 1997. Diagnosis and treatment of disease caused by nontuberculous mycobacteria. Am J Respir Crit Care Med 156: 1-25.

Bouley DM, Ghori N, Mercer KL, Falkow S, Ramakrishnan L 2001. Dynamic nature of host-pathogen interactions in Mycobacterium marinum granulomas. Infect Immun 69: 7820-7831.

Brennan PJ 2003. Structure, function and biogenesis of the cell wall of Mycobacterium tuberculosis. Tuberculosis (Edinb) 83: 91-97.

Brown-Elliott BA, Wallace RJ Jr 2005. Infections caused by nontuberculous mycobacteria. In GL Mandell, JC Bennett, R Dolin (eds.), Mandell, Douglas and Bennett's: Principles and practice of infectious diseases, Vol. 2, 6th ed., Elsevier, Philadelphia, p. 2909-2916.

Chan ED, Bai X, Kartalija M, Orme IM, Ordway DJ 2010. Host immune response to rapidly growing mycobacteria, an emerging cause of chronic lung disease. Am J Respir Cell Mol Biol: in press.

Chatterjee D, Khoo KH 1998. Mycobacterial lipoarabinomannan: an extraordinary lipoheteroglycan with profound physiological effects. Glycobiology 8: 113-120.

Da Silva TR, De Freitas JR, Silva QC, Figueira CP, Roxo E, Leão SC, De Freitas LA, Veras PS 2002. Virulent Mycobacterium fortuitum restricts NO production by a gamma interferon-activated $\mathrm{J} 774$ cell line and phagosome-lysosome fusion. Infect Immun 70: 5628-5634.

Denis M, Forget A, Pelletier M, Turcotte R, Skamene E 1986. Control of Bcg gene of early resistance in mice to infections with BCG substrains and atypical mycobacteria. Clin Exp Immunol 63: 517-525.

Flynn JL, Scanga CA, Tanaka KE, Chan J 1998. Effects of aminoguanidine on latent murine tuberculosis. J Immunol 160: 1796-1803.

Griffith DE, Aksamit T, Brown-Elliott BA, Catanzaro A, Daley C, Gordin F, Holland SM, Horsburgh R, Huitt G, Iademarco MF, Iseman M, Olivier K, Ruoss S, von Reyn CF, Wallace RJ Jr, Winthrop K 2007. An official ATS/IDSA statement: diagnosis, treatment and prevention of nontuberculous mycobacterial diseases. Am J Respir Crit Care Med 175: 367-416. 
Hänsch HC, Smith DA, Mielke ME, Hahn H, Bancroft GJ, Ehlers S 1996. Mechanisms of granuloma formation in murine Mycobacterium avium infection: the contribution of $\mathrm{CD}^{+} \mathrm{T}$ cells. Int Immunol 8: 1299-1310.

Hein J, Schellenberg U, Bein G, Hackstein H 2001. Quantification of murine IFN-gamma mRNA and protein expression: impact of real-time kinetic RT-PCR using SYBR green I dye. Scand J Immunol 54: 285-291.

Ho MH, Ho CK, Chong LY 2006. Atypical mycobacterial cutaneous infections in Hong Kong: 10-year retrospective study. Hong Kong Med J 12: 21-26

Hogan LH, Macvilay K, Barger B, Co D, Malkovska I, Fennelly G, Sandor M 2001. Mycobacterium bovis strain bacillus CalmetteGuérin-induced liver granulomas contain a diverse TCR repertoire, but a monoclonal $\mathrm{T}$ cell population is sufficient for protective granuloma formation. J Immunol 166: 6367-6375.

Jarzembowski JA, Young MB 2008. Nontuberculous mycobacterial infections. Arch Pathol Lab Med 132: 1333-1341.

Khalil A, Bourut C, Halle-Pannenko O, Mathé G, Rappaport H 1975. Histologic reactions of the thymus, spleen, liver and lymph nodes to intravenous and subcutaneous BCG injections. Biomedicine 22: 112-121.

Ladel CH, Daugelat S, Kaufmann SH 1995. Immune response to Mycobacterium bovis bacille Calmette Guérin infection in major histocompatibility complex class I and II-deficient knock-out mice: contribution of CD4 and CD8 T cells to adquired resistance. Eur J Immunol 25: 377-384.

MacMicking JD, North RJ, LaCourse R, Mudgett JS, Shah SK, Nathan CF 1997. Identification of nitric oxide synthase as a protective locus against tuberculosis. Proc Natl Acad Sci USA 94: 5243-5248.

Mohan VP, Scanga CA, Yu K, Scott HM, Tanaka KE, Tsang E, Tsai MM, Flynn JL, Chan J 2001. Effects of tumor necrosis factor alpha on host immune response in chronic persistent tuberculosis: possible role for limiting pathology. Infect Immun 69: 1847-1855.

Nigou J, Gilleron M, Rojas M, García LF, Thurnher M, Puzo G 2002. Mycobacterial lipoarabinomannans: modulators of dendritic cell function and the apoptotic response. Microbes Infect 4: 945-953.

Orrell JM, Brett SJ, Ivanyi J, Coghill G, Grant A, Beck JS 1992. Mor- phometric analysis of Mycobacterium tuberculosis infection in mice suggests a genetic influence on the generation of the granulomatous inflammatory response. J Pathol 166: 77-82.

Palwade PK, Dhurat RS, Tendolkar UM, Dethe GR, Jerajani HR 2006. Chronic cutaneous disease caused by the rapid growers Mycobacterium fortuitum and chelonae. Br J Dermatol 154: 774-775.

Parti RP, Srivastava S, Gachhui R, Srivastava KK, Srivastava R 2005. Murine infection model for Mycobacterium fortuitum. Microbes Infect 7: 349-355.

Runyon EH 1959. Anonymous mycobacteria in pulmonary disease. Med Clin North Am 43: 273-290.

Russell DG, Mwandumba HC, Rhoades EE 2002. Mycobacterium and the coat of many lipids. J Cell Biol 158: 421-426.

Sato IY, Kobayashi K, Kasama T, Kaga S, Kasahara K, Kanemitsu H, Nakatani K, Takahashi T, Nakamura RM, Skamene E, Yoshida T 1990. Regulation of Mycobacterium bovis BCG and foreign body granulomas in mice by the Bcg gene. Infect Immun 58: 1210-1216.

Sungkanuparph S, Sathapatayavongs B, Pracharktam R 2003. Rapidly growing mycobacterial infections: spectrum of diseases, antimicrobial susceptibility, pathology and treatment outcomes. J Med Assoc Thai 86: 772-780.

Uslan DZ, Kowalski TJ, Wengenack NL, Virk A, Wilson JW 2006. Skin and soft tissue infections due to rapidly growing mycobacteria: comparison of clinical features, treatment and susceptibility. Arch Dermatol 142: 1287-1292.

van der Sar AM, Abdallah AM, Sparrius M, Reinders E, Vandenbroucke-Grauls CM, Bitter W 2004. Mycobacterium marinum strains can be divided into two distinct types based on genetic diversity and virulence. Infect Immun 72: 6306-6312.

Vercellone A, Nigou J, Puzo G 1998. Relationships between the structure and the roles of lipoarabinomannans and related glycoconjugates in tuberculosis pathogenesis. Front Biosci 3: 149-163.

Weitzul S, Eichhorn PJ, Pandya AG 2000. Nontuberculous mycobacterial infections of the skin. Dermatol Clin 18: 359-377.

Zhu G, Xiao H, Mohan VP, Tanaka K, Tyagi S, Tsen F, Salgame P, Chan J 2003. Gene expression in the tuberculous granuloma: analysis by laser capture microdissection and real-time PCR. Cell Microbiol 5: 445-453. 